\title{
THE AFRICAN CARIBBEAN EYE SURVEY: RISK FACTORS FOR GLAUCOMA IN A SAMPLE OF AFRICAN CARIBBEAN PEOPLE LIVING IN LONDON
}

\author{
R. P. L. WORMALD, E. BASAURI, L. A. WRIGHT and J. R. EVANS \\ London
}

\begin{abstract}
SUMMARY
The purpose of the study was to estimate the prevalence of and risk factors for chronic glaucoma in a sample of African Caribbean people over 35 years of age living in the London Borough of Haringey. A cross-sectional voluntary sample of persons were subjected to detailed ophthalmic assessment including automated tangent screen suprathreshold visual field testing, applanation tonometry and stereoscopic disc evaluation in $50 \mathrm{com}-$ munity-based survey clinics over an 8 month period. Cases and suspects were referred to Moorfields Eye Hospital for more detailed assessment and confirmation of the diagnosis. Of 873 eligible persons examined (out of a total of 1022), 32 definite cases of glaucoma were identified, a prevalence of $3.9 \% ; 42 \%$ of these had been previously diagnosed. Approximately $10 \%$ of the sample required further assessment and follow-up when ocular hypertensives and glaucoma suspects were included. An age-standardised comparison with the findings of the Roscommon survey revealed a relative risk for glaucoma for Haringey blacks compared with Irish whites of 3.7. Significant risk factors for glaucoma included age, African birthplace and darker skin colour. Neither diabetes nor hypertension reached significance. Despite the lack of a population base, this study provides strong evidence that the 4 times greater risk of glaucoma estimated for American blacks compared with whites applies equally to the United Kingdom population. Community-based facilities are required to raise awareness of the risk among this ethnic minority in this country and casefinding resources should be provided to meet local needs.
\end{abstract}

There is growing evidence that people of African origin are at increased risk of blinding eye disease, especially glaucoma. ${ }^{1-4}$ Because of this, a survey of eye health among London's immigrant African and African-Carib-

From: Department of Preventive Ophthalmology, Institute of Ophthalmology, University of London, London, UK.

Correspondence to: Mr Richard Wormald, Unit of Ophthalmology,

Western Eye Hospital, Marylebone Road, London NW1 5 YE, UK. bean community was planned in order to discover whether similar risks exist for this ethnic group in the United Kingdom (UK). Statistics from the United States ${ }^{5,6}$ indicate that glaucoma is the most common cause of blind registration for American blacks. No data on ethnicity is available in the UK blind and partial sight registers and no other sources of epidemiological data on the eye health of this ethnic minority exist in the UK. It was therefore felt that such a survey was an urgent necessity. The main objectives of the study were:

1. To estimate the prevalence of glaucoma and other common blinding eye diseases in UK blacks.

2. To identify risk factors for blinding eye disease in this community.

3. To increase awareness of eye health issues in the black community, especially glaucoma risk.

The authors of the Baltimore Eye Survey ${ }^{1.4}$ estimate that American blacks have approximately a 4 times greater risk of chronic open angle glaucoma than American whites. The survey in St Lucia ${ }^{7}$ (of the eye health of the black population) and the Barbados Eye Study ${ }^{8.9}$ (pilot study results) estimate prevalences of $8.8 \%$ (30 years of age and over) and $13 \%$ (35 years and over) respectively for the black populations. While there are few strictly populationbased reports from West Africa, it appears that glaucoma is very common in some areas but less so in others. ${ }^{10.11}$ Bartholomew ${ }^{12}$ found no evidence for an increased glaucoma prevalence in the Pondo tribe of the Transvaal.

The black population in the UK is largely of Jamaican origin although there are significant numbers from other Caribbean islands and Africa. In this survey $84 \%$ of the sample were from Caribbean islands (of whom $72 \%$ were from Jamaica), and $16 \%$ were from Africa. The Jamaicans and other Caribbean islanders came to the UK around 30 years ago while the African immigrants tend to be more recent arrivals. In this study the mean length of stay in the UK for Caribbeans was 29 years while it was 16 years for

Eye (1994) 8, 315-320 C 1994 Royal College of Ophthalmologists 
Africans. It is said that Jamaicans are genetically more ethnically mixed (similar to American blacks) than other Caribbean islanders.

This paper reports on the findings of the survey with regard to glaucoma prevalence and estimates of relative risk for glaucoma within this group. Exposures of interest included those already established as risk factors for glaucoma including age, sex, family history, diabetes and hypertension. In addition we considered skin colour, which was found to be associated with intraocular pressure in Barbados, ${ }^{8.9}$ and country of origin.

\section{PARTICIPANTS AND DESIGN}

The London Borough of Haringey has one of the highest proportions of ethnic minorities in the country, including a large population of African and Caribbean people numbering 27500 in the 1991 census (approximately 15\% of the total Haringey population). Of these, $37 \%$ are over the age of 35 years ( 10125 people). Both the local health authority and the borough council backed the project by providing logistical support. Meetings were held with local community leaders in order to encourage participation and to raise the level of awareness within the community with regard to eye health issues. Despite these measures and extensive publicity in the black press and radio, insufficient resources were available to the study to raise a population-based random sample.

Originally, residential addresses in randomly sampled postcodes from borough wards where the highest concentration of African Caribbean people were living at the time of the 1981 census were visited by members of the study team or local volunteer support groups. Any person aged 35 years and over, living in the chosen postcode areas, having parents born in Africa or the Caribbean, and considering him/herself belonging to that ethnic group, was invited to participate in the survey.

This method achieved no more than a $40 \%$ response rate, which could not be improved despite redoubled efforts from the study team and volunteers. A second strategy using local church and community organisations as ad hoc sampling frames also failed to achieve a response better than $50 \%$. Ultimately, it was necessary to accept that the best that could be achieved given available resources was a voluntary sample without a population-based denominator.

Community-based survey clinics at six different sites in Haringey were well attended. Fifty such clinics were held over an 8 month period (April-December 1991). At each clinic, after subjects had given their informed consent, background information on personal and family medical and ocular history, migration history and other routine demographic covariates were gathered on standard proformata. Blood pressure and random blood sugar were measured by a qualified nurse. Sickledex was also available for those who wished to be tested for sickle cell disease. A full ophthalmological examination was then performed including binocular and uniocular visual acuity (best corrected and with pinhole if $<6 / 9$ ), near vision (N6 with either eye) Takagi Automated Tangent Screen (ATS) central $25^{\circ}$ static suprathreshold visual fields, applanation tonometry, post-mydriatic ( $1 \%$ tropicamide) grading of lens opacity (LOCS II), vertical cup-to-disc ratio (CDR) and narrowest neuroretinal rim (NNR) and, where possible, non-simultaneous stereo fundus photography using a Canon CR4 'non-mydriatic' camera with Ektachrome film.

Gonioscopy was not routinely performed in the survey clinics except when the peripheral angle appeared shallow according to the method described by Van Herick et al. ${ }^{13}$ This was because contact lens examination was not well tolerated by participants and interfered with the optical surface of the cornea and hence the quality of the fundus photographs.

\section{Case Definitions}

Glaucoma: Already diagnosed as glaucoma (confirmed at survey) and receiving treatment or newly diagnosed during the survey and confirmed on follow-up assessment at Moorfields Eye Hospital on agreement by two consultant ophthalmologists. The sine qua non for this definition was the presence of a repeatable glaucomatous visual field defect in one or both eyes or visual loss so advanced as to preclude field testing attributable to glaucoma by both ophthalmologists in one or both eyes.

Glaucoma Suspect: Presence of abnormal optic disc appearance and/or visual field defect with or without raised intraocular pressure (IOP) but unconfirmed as a definite case on follow-up.

Ocular hypertensive: IOP consistently over $21 \mathrm{mmHg}$ in one or both eyes but with no other sign of glaucoma.

All cases, suspects and ocular hypertensives were referred to a special clinic at Moorfields where standardised assessment took place including gonioscopy of both eyes and Humphrey threshold 24-2 visual field analysis.

\section{Definition of Exposures}

Diabetes: A positive history of diabetes on treatment including diet alone, tablets and insulin dependence or a random blood sugar tested on the Glucocheck device with BM sticks above $15 \mathrm{mmol}$.

Hypertension: A positive history of current therapy for hypertension and/or a measured diastolic blood pressure of $>100 \mathrm{mmHg}$ sitting using an automatic Takea sphygmomanometer.

Skin colour: Skin colour was graded by one of two ophthalmologists as light, medium or dark. Light skin colour was defined as unusually light for this ethnic group and dark as noticeably darker than average. Whilst these gradings were subjective, they were based on simple observation with good informally assessed concordance between the two observers.

Family history: These data were not analysed because they were felt to be of poor reliability. This was due to lack of up-to-date health information on relatives being available to participants and, frequently, unfamiliarity with the 
term glaucoma, so that if there was a history of blindness. its cause could not be determined with any certainty.

Country of origin: This was based on the participant's stated place of birth.

\section{Statistical Methods}

Data were entered onto a microcomputer using Paradox and were analysed in SAS ${ }^{14}$ and Egret. ${ }^{15}$ The crude association between risk factors and glaucoma was assessed by calculating risk ratios. Pearson's chi-squared test was used to test whether the observed risk ratios were significantly different from 1 at the $5 \%$ level. A logistic regression model was used to analyse the effect of the risk factors together, the measure of effect being the odds ratio. The model included terms for age $(35-49,50-59$ and $60+$ years), sex, diabetes, hypertension, skin colour, and place of birth of self or parents.

Three different outcomes as defined above were modelled: (1) definite glaucoma. (2) definite glaucoma + glaucoma suspects and (3) the above + ocular hypertensives.

Data for both univariate and multivariate analysis were not always complete for certain exposures so that only those subjects for whom all required exposures were recorded have been included in the analysis. Thus some variation in the numerator and denominator will be observed for different exposures in Tables I and II, only subjects for whom data on all exposures were available being included.

\section{RESULTS}

A total of 1022 people were examined in the survey clinics. Of these, 873 were African Caribbean and over 35 years of age. While some of the attenders were 'sampled', the majority were voluntary attenders coming to the survey clinics on the recommendation of friends or relatives

Table I. Cross-tabulation of glaucoma cases (definite) by various exposures

\begin{tabular}{|c|c|c|c|c|}
\hline Risk factor & $n$ & No. of cases & c/c with glaucoma & $p$ value $\left(\chi^{2}\right)$ \\
\hline \multicolumn{5}{|l|}{ Age (years) } \\
\hline$<50$ & 256 & 3 & 1.2 & \multirow[t]{3}{*}{$<0.001$} \\
\hline $50-59$ & 299 & 3 & 1.0 & \\
\hline $60+$ & 318 & 26 & 8.2 & \\
\hline \multicolumn{5}{|l|}{ Sex } \\
\hline Male & 313 & 11 & 3.5 & \multirow[t]{2}{*}{0.86} \\
\hline Female & 560 & 21 & 3.8 & \\
\hline \multicolumn{5}{|c|}{ Hypertension" } \\
\hline Yes & 365 & 13 & 3.6 & \multirow[t]{2}{*}{0.91} \\
\hline No & 497 & 17 & 3.4 & \\
\hline \multicolumn{5}{|l|}{ Diabetes" } \\
\hline Yes & 115 & 9 & 7.8 & \multirow[t]{2}{*}{0.009} \\
\hline No & 742 & 22 & 3.0 & \\
\hline \multicolumn{5}{|l|}{ Skin colour ${ }^{a}$} \\
\hline Light & 176 & 3 & 1.7 & \multirow[t]{3}{*}{0.38} \\
\hline Medium & 564 & 20 & 3.5 & \\
\hline Dark & 88 & 4 & 4.5 & \\
\hline \multicolumn{5}{|c|}{ Place of birth } \\
\hline Jamaica & 523 & 17 & 3.3 & \multirow[t]{3}{*}{0.58} \\
\hline Other & 214 & 8 & 3.7 & \\
\hline Africa & 136 & 7 & 5.1 & \\
\hline
\end{tabular}

"Missing data. or as a result of the publicity campaign. Thus a response rate could not be calculated. In all, 50 clinics with an average response of 20 per clinic were performed (range 2-82). Each participant spent an average of 90 minutes in the clinic. Distribution of age and sex of the sample and other risk factors is illustrated in Fig. 1.

The prevalence of visual impairment by WHO criteria $(<6 / 18$ best vision in the better-seeing eye or gross visual field constriction to $<10^{\circ}$ ) was $2.3 \%$ (20 persons). Forty per cent of this was due to cataract and only 1 case was due to glaucoma. Only 2 blind persons $(<3 / 60)$ attended the survey, 1 of whom was blind due to diabetic eye disease and the other due to bilateral macula disease. One hundred and thirteen persons were referred for further glaucoma assessment. After 2 years, 24 persons persistently failed follow-up appointments despite many efforts to contact them. Thirty-three cases of definite glaucoma were identified of which 14 were previously diagnosed (42\%) and under the care of an ophthalmologist and therefore not referred. It was possible to confirm all these cases as glaucoma on the basis of clinical findings in the survey clinic. The prevalence of definite glaucoma was $3.9 \%$ in this sample. A further 37 persons were glaucoma suspects and 19 were ocular hypertensives, 8 of the latter being already under the hospital eye service. A total of 87 individuals (prevalence $10.1 \%$ ) were found to require further assessment or follow-up for glaucoma. The remaining 24 persons who were referred were found to be normal on assessment at Moorfields Eye Hospital. These were mostly low-risk ocular hypertensives, persons with slight disc asymmetry or minimal field loss who nevertheless were referred according to the study protocol. Fig. 2 illustrates these prevalences.

Univariate analysis of the risk factors for glaucoma described above are tabulated in Table I. The results of logistic regression analysis are shown in Table II. In these three models, increasing age, darker skin and a place of origin in Africa are associated with a significantly increased risk of disease. Diabetes and hypertension do not feature as significant risk factors.

\section{DISCUSSION}

This study provided important experience of sampling problems in an urban environment and, in particular, the difficulty of accessing a specific ethnic minority group. Similar difficulties were experienced during the Baltimore Eye Survey ${ }^{16}$ although these were overcome using professional sampling agents and intensive community-based resources. In the Baltimore Eye Survey a household was not classified as non-responding until 10 attempts to reach the occupants had failed. Such a protocol was beyond the scope of resources available for this study. We felt that despite these difficulties, it was worth pursuing the investigation.

As a volunteer sample, it is likely that there was a 'healthy volunteer effect'. It is reasonable to assume that severely visually impaired persons who have already been assessed and diagnosed are unlikely to attend such a sur- 
Table II. Multivariate risk factor analysis for glaucoma. suspects and ocular hypertension

\begin{tabular}{|c|c|c|c|c|c|c|}
\hline \multirow[b]{2}{*}{ Risk factor } & \multicolumn{2}{|c|}{ Model 1} & \multicolumn{2}{|c|}{ Model 2} & \multicolumn{2}{|c|}{ Model 3} \\
\hline & Odds ratio & $95 \%$ CI & Odds ratio ${ }^{a}$ & $95 \% \mathrm{CI}$ & Odds ratio" & $95 \% \mathrm{CI}$ \\
\hline \multicolumn{7}{|l|}{ Age (years) } \\
\hline$<50$ & 1 & & 1 & & 1 & \\
\hline $50-59$ & 0.6 & $0.06-6.1$ & 2.4 & $1-6.1$ & 2 & $0.9-4.2$ \\
\hline $60+$ & 15.1 & $3.3-69.6^{*}$ & 5.2 & $2.1-12.7 *$ & 3.9 & $1.8-8.3^{*}$ \\
\hline \multicolumn{7}{|l|}{ Sex } \\
\hline Male & 1 & & 1 & & 1 & \\
\hline Female & 1.2 & $0.5-2.8$ & 1.3 & $0.7-2.3$ & 1.1 & $0.7-1.8$ \\
\hline \multicolumn{7}{|l|}{ Diabetes } \\
\hline No & 1 & & 1 & & 1 & \\
\hline Yes & 2.2 & $0.9-5.6$ & 1.4 & $0.7-2.8$ & 1.2 & $0.6-2.3$ \\
\hline \multicolumn{7}{|c|}{ Hypertension } \\
\hline No & 1 & & 1 & & 1 & \\
\hline Yes & 0.9 & $0.4-2$ & 0.6 & $0.4-1.1$ & 0.9 & $0.5-1.4$ \\
\hline \multicolumn{7}{|l|}{ Skin colour } \\
\hline Light & 1 & & 1 & & 1 & \\
\hline Medium & 2.1 & $0.6-7.6$ & 1.7 & $0.8-3.8$ & 1.6 & $0.8-3.1$ \\
\hline Dark & 2.2 & $0.4-12.3$ & 2.7 & $1-7.8^{*}$ & 3.2 & $1.3-7.6^{*}$ \\
\hline \multicolumn{7}{|c|}{ Place of birth } \\
\hline Jamaica & 1 & & 1 & & 1 & \\
\hline Africa & 6.7 & $1.8-24.7 *$ & 1.8 & $0.7-4.2$ & 1.6 & $0.8-3.5$ \\
\hline Other & 1.1 & $0.4-3.1$ & 0.7 & $0.4-1.5$ & 0.8 & $0.4-1.5$ \\
\hline
\end{tabular}

CI, confidence interval. $*$ Significant at the 0.05 level.

"Taken from logistic regression model with terms for all risk factors in table.

Model 1 outcome $=$ definite glaucoma; model 2 outcome $=$ definite glaucoma and glaucoma suspects; model 3 outcome $=$ definite glaucoma, glaucoma suspects and ocular hypertensives.

vey clinic on a voluntary basis. It is significant that so few of the visually impaired were suffering from glaucoma when we know that glaucoma is the commonest cause of blind registration for blacks in the United States. These data are therefore unlikely to be representative of the community as a whole. Although it is doubtful that significant bias is likely to affect response/non-response for asymptomatic and previously undiagnosed glaucoma, it is likely that patients with known glaucoma would not attend being already under the care of eye specialists. This might explain the higher ratio of newly diagnosed disease to established disease. In almost all recent surveys, including the Baltimore Eye Survey, ${ }^{1.4}$ this ratio is $1: 1$, while in this study it was 1.3:1 newly diagnosed to established disease.

Also important is the age structure of the population sample. There is a marked tailing off in the older age groups which is due in part to the age at migration to the

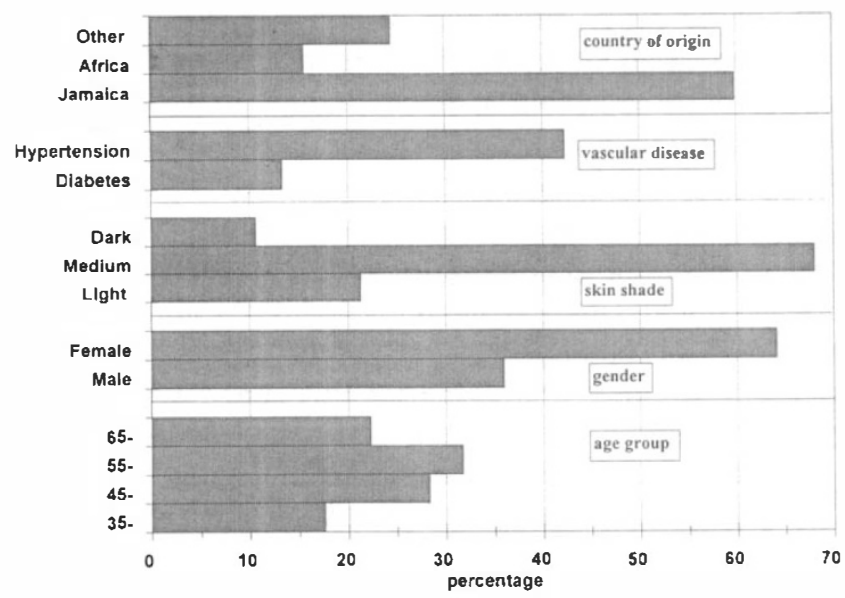

Fig. 1. Distribution of risk factors in the study population.
UK and in part to the fact that some West Indians return to the Caribbean after retirement. In addition to this, there may be a tendency for the elder members of the community not to attend because of infirmity from other causes. The crude prevalence of glaucoma for participants over 50 years of age was $4.7 \%$ compared with $1.8 \%$ as estimated in a recently published survey from the West of Ireland, ${ }^{17}$ a relative risk of 2.6 for blacks. But after direct age standardisation, using age-specific rates from this study and the population structure of Roscommon, the adjusted prevalence becomes $6.9 \%$ and the relative risk for blacks 50 years and over compared with the white population in the West of Ireland is 3.7. This relative risk is comparable with that found in Baltimore and, because of

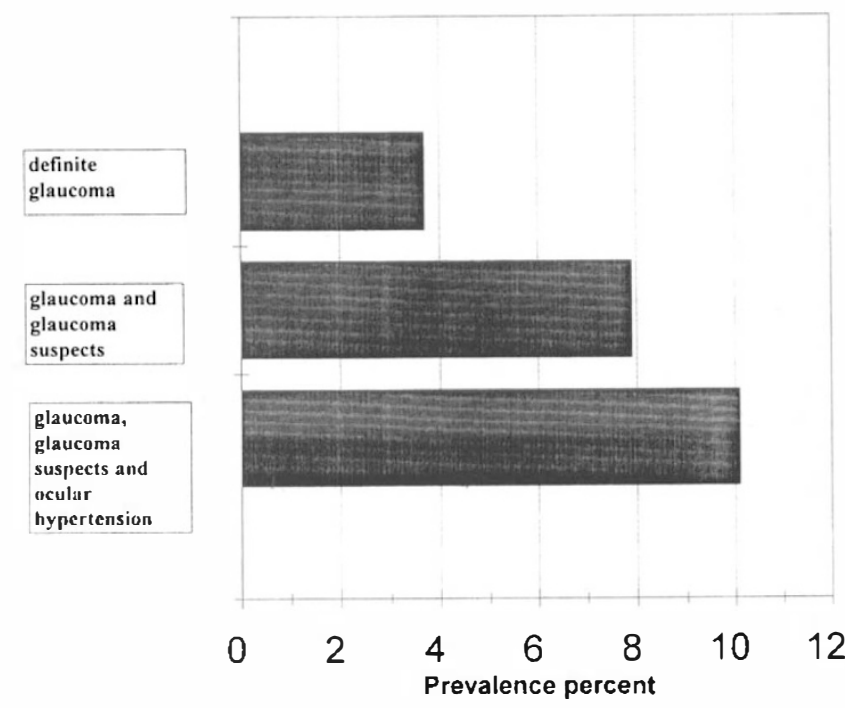

Fig. 2. Prevalence of glaucoma, suspect glaucoma and ocular hypertension. 
reasons already stated, may well underestimate the true relative risk.

Assuming the sample of glaucoma cases found in this survey is biased by being previously undetected and probably under-representing the older age groups, the influence of this bias on the validity of the multivariate risk factor analysis requires consideration for each risk factor assessed.

\section{Age}

Because the age structure of this population subgroup in the UK is itself skewed and there were so few participants from the older age groups in the study, the effect of the aforementioned presumptive biases in attendance on the observed relative risks for increasing age are difficult to estimate. If there was an interaction between increasing age and being a glaucoma sufferer on the likelihood of not attending, then it is likely that the relative risk attributed to increasing age has been underestimated. The finding of a reduced risk for the middle age band for glaucoma model 1 (Table II) indicates that compared with the younger age band (used as baseline risk) the risk is lower (although not significantly so). This might be explained by there being more diagnosed disease in the middle age band which did not attend, or it may possibly reflect a biphasic risk of disease by age in blacks which has been alluded to in a small case series from Africa. ${ }^{17}$

\section{Sex}

No effect was seen and more women than men attended the survey. Other studies have reported a male predominance, especially from Africa, and this effect may have been missed because of affected males not attending the survey.

\section{Diabetes and Hypertension}

It is interesting that neither diabetes nor hypertension appears as a significant risk in any model, although diabetes does reach significance in the univariate analysis and is close to significance in the model for definite glaucoma as an outcome alone. The fact that the univariate association is lost in the model suggests that much of this observed effect was confounded by another variable, probably age, i.e. the risk of glaucoma increases with age as does the risk of diabetes.

The Baltimore Eye Survey has recently reported a similar finding except for a weak association with systolic blood pressure. ${ }^{18}$ It is possible that a greater proportion of diagnosed patients have either or both these diseases because the diagnosis of either condition should indicate an eye examination and consequent identification of glaucoma - in which case, an under-representation of previously diagnosed disease would lead to fewer associated cases with either diabetes and/or hypertension. However, the prevalence of both these conditions was high in the sample by the definitions given above (Fig. 1). There was therefore ample opportunity for such an association, if present, to be identified. It is possible that the previously observed associations arise from hospital-based selection bias: i.e. patients with either condition are more likely to have their eyes examined and glaucoma identified.

\section{Skin Pigmentation}

The interesting finding regarding skin pigmentation corresponds to a reported association between skin colour and IOP in the Barbados Eye Study. It is also compatible with the theory that the degree of ocular pigmentation is implicated in the pathogenesis of glaucoma beyond pigmentary glaucoma in the pigment dispersion syndrome. It is hard to imagine how skin pigmentation (which was always graded before ocular assessment) may have biased attendance or glaucoma diagnosis when the final diagnosis of glaucoma was made at Moorfields, independent of skin colour grading. However, the relative risk only reaches significance for the dark grade in model 3 when ocular hypertensives are included. It may simply reflect a consistency with the findings of the Barbados study, i.e. the association is with IOP rather than glaucoma per se. While there was a trend, there was no significant difference in the mean IOP for right or left eyes by skin colour (Table III).

\section{Africa}

Finally, the finding regarding African origin, which is the strongest odds ratio after age for model 1 for definite glaucoma only, is most interesting. Africans accounted for only $16 \%$ of the sample and were younger than the West Indians. They came mainly from Nigeria and Ghana. The response bias for previously undiagnosed cases may be relevant here because they are more recent immigrants with possibly poorer access to health services and a lower awareness of glaucoma risk. The data from this study are insufficient to throw more light on this observation and it should probably be interpreted with caution. Both old ${ }^{19}$ and recent work from Africa suggests that the distribution of glaucoma in West Africa is far from uniform. Further population-based research in West Africa is urgently needed to address this question.

Nineteen new cases of glaucoma were identified in the survey and only 5 of these $(26 \%)$ had an IOP greater than $21 \mathrm{mmHg}$ in one or both eyes when first tested in the survey clinics. This proportion is lower even than was found in the Baltimore Eye Survey, where half the new cases were normotensive at survey. It is possible that the proportion of normotensive glaucoma is higher in blacks than whites, although this was not observed in Baltimore. In Roscommon, 13 of the 28 newly diagnosed cases had raised IOP in the survey clinics (46\%). This finding reinforces the vitally important concept of IOP as a risk factor for glaucoma rather than a sine qua non for diagnosis.

Table III. Intraocular pressure (IOP) in relation to skin colour

\begin{tabular}{lcccc}
\hline Skin colour & $n$ & IOP (max.) & IOP right eye & IOP left eye \\
\hline Light & 171 & $16.2 \pm 2.9$ & $16.0 \pm 3.6$ & $15.6 \pm 3.5$ \\
Medium & 546 & $16.8 \pm 3.4$ & $15.9 \pm 4.0$ & $15.9 \pm 4.0$ \\
Dark & 87 & $17.1 \pm 3.9$ & $16.1 \pm 3.8$ & $16.5 \pm 4.1$ \\
\hline
\end{tabular}


Although this study failed to achieve the random sample of the population which would justify the application of sampling theory and the calculation of confidence limits around the estimates of prevalence, it does provide evidence that the relative risk of glaucoma in blacks in the UK compared with whites is at least as great as that found in Baltimore. The lack of association with diabetes and hypertension is also relevant, especially as this parallels the Baltimore Eye Survey finding and indicates that the increased risk of glaucoma in blacks cannot easily be explained by the greater prevalence of these two conditions in this ethnic minority.

This work should serve as a focus to help raise the awareness of the problem among both clinicians and the African Caribbean population in this country, so that they can be encouraged to seek case-finding facilities in the community before their disease reaches such an advanced stage that it becomes symptomatic.

The authors gratefully acknowledge help and support from the following. Steering and support: Bernie Grant, MP: Gladwell Msimang; Haringey Health Authority: Dr Keyvan Zahir; Haringey Council Social Services: Olsen Samuels and Henderson Holmes; Haringey Council: Councillor Errol Neckles and Gurbux Singh. Funding: the RNIB, the Wolfson Foundation, IGA, Council of Churches for Britain and Ireland. Centres: The West Indian Centre, Broadwater Farm Residents Association, St Ann's Hospital George Marsh Centre, the Wellbourne Community Centre, Whitehall and Tenterton Community Centre, Lordship Lane clinic, and all the support staff and helpers at these centres. All the staff and volunteers at the clinics including: Jennifer Lee, Hildreth McPherson, Chris Steele, Pak San Lee, Hugh Lugg, Amos, and the orthoptists and nurses from Moorfields Eye Hospital.

Key words: Afro-Caribbean, Epidemiology, Ethnic minority, Glaucoma, Risk factors, Visual impairment.

\section{REFERENCES}

1. Teilsch JM, Sommer A, Katz J, et al. Racial variations in the prevalence of primary open-angle glaucoma: the Baltimore Eye Survey. JAMA 1991;266:369-74.

2. Morton Grant W, Burke JF. Why do some people go blind from glaucoma? Ophthalmology 1982;89:991-8.
3. Wilensky JT, Gandhi N, Pan T. Racial influences in openangle glaucoma. Ann Ophthalmol 1978;10:1398-402.

4. Tielsch JM, Sommer A, Witt K, et al. Blindness and visual impairment in an American urban population: the Baltimore Eye Survey. Arch Ophthalmol 1990;108:286-90.

5. Hiller R, Kahn HA. Blindness from glaucoma. Am J Ophthalmol 1975;80:62-9.

6. Greenbridge KC, Dweck M. Glaucoma in the black population: a problem of blindness. J Natl Med Assoc 1988:80:1305-9.

7. Mason RP, Kosoko O, Wilson R, Martone JF, Cowan CI, Gear JC, et al. National survey of the prevalence of glaucoma in St Lucia, West Indies. Ophthalmology 1989; 96:1363-8.

8. Leske MC, Connel AMS, Kehoe R. A pilot project of glaucoma in Barbados. Br J Ophthalmol 1989;73:365-9.

9. Leske MC, Troutman HT. Connel A. Glaucoma in Barbados. Arch Ophthalmol 1989;107:169.

10. Murdoch I, Babalola O, Abiose A, Jones B. Pattern of intraocular pressure and cup-disc ratio and prevalence of glaucoma in Hausa/Fulani rural communities in northern Nigeria. Invest Ophthalmol Vis Sci 1993;34(Suppl):2914.

11. Faal H, Minassian D, et al. National survey of blindness and low vision in the Gambia. Br J Ophthalmol 1989;73:82-7.

12. Bartholemew RS. Glaucoma in a South African black population: a population study of the Pondo tribe of South Africa. S Afr Arch Ophthalmol 1976;3:135-48.

13. Van Herick W, Shaffer RN, Schwartz A. Estimation of width of angle of anterior chamber. Am J Ophthalmol 1969; 68:626-9.

14. SAS language guide for personal computers. Cary, NC: SAS Institute, 1988.

15. Egret Statistics and Epidemiology Research Corporation, Seattle, Washington, 1988.

16. Baltimore Eye Survey, manual of operations. Baltimore, Maryland: International Centre for Epidemiology and Preventive Ophthalmology, John Hopkins University, 1985.

17. Coffey M, Reidy A, Wormald R. Wu XX. Wright L, Courtney P. Prevalence of glaucoma in the west of Ireland. Br J Ophthalmol 1993;77:17-21.

18. Tielsch JM, Katz J, Javitt J, Quigley HA, Sommer A. Systemic risk factors for primary open-angle glaucoma. Invest Ophthalmol Vis Sci 1993;34(Suppl):971.

19. Verry J-D, Foster A, Wormald R, Akuamoa C. Chronic glaucoma in northern Ghana: a retrospective study of 397 patients. Eye 1990;4:115-20. 